\title{
A new wake-up radio architecture for wireless sensor networks
}

\author{
Florin Hutu*, Aissa Khoumeri, Guillaume Villemaud and Jean-Marie Gorce
}

\begin{abstract}
The last decades have been really hungry in new ways to reduce energy consumption. That is especially true when talking about wireless sensor networks in general and home multimedia networks in particular, since electrical energy consumption is the bottleneck of the network. One of the most energy-consuming functional block of an equipment is the radio front end, and methods to switch it off during the time intervals where it is not active must be implemented. This paper proposes a wake-up radio circuit which is capable of both addressing and waking up not only a more efficient but also more energy-consuming radio front end. By using a frequency footprint to differentiate each sensor, awaking all the sensors except for the one of interest is avoided. The particularity of the proposed wake-up receiver is that the decision is taken in the radio-frequency part and no baseband treatment is needed.
\end{abstract}

Keywords: Wake-up radio; Energy consumption; Wireless sensor networks

\section{Introduction}

The tremendous soar of the telecommunications domain was accompanied by a directly proportional increase of electrical energy consumption. Often, performance criteria such as spectral efficiency or data rate are optimized at the expense of more energy consumption. Recent advances in analogue and digital electronics have enabled the development of low-cost, low-energy wireless nodes. However, energy consumption remains the crucial characteristic as it determines the entire network lifetime. Idle mode listening, typically used by sensors in this kind of networks, impacts the overall energy consumption. Nevertheless, this can be particularly suboptimal when the data traffic is very sporadic.

Wireless sensor networks (WSNs) have their place in various applications like environmental monitoring applications [1,2], in which WSNs provide various information, such as temperature, air pressure, and humidity. WSNs are also used in various domains like noise monitoring, military applications, control, battlefield surveillance, home networking, etc. This area is particularly important on one hand because the energy consumption of home multimedia equipment represents an important part of the overall energy consumption. On the other hand, it is important

\footnotetext{
*Correspondence: florin-doru.hutu@insa-lyon.fr INRIA, INSA-Lyon, CITI-INRIA, Université de Lyon, 6, avenue des Arts, F-69621 Villeurbanne, Lyon, France
}

because there is a lack of methods to optimize the energy consumption of these types of equipment. The focus of this paper is reduction of energy consumption of wireless equipment. Energy reduction is performed by proposing a system which is able to switch off the radio frequency interface during the inactive period and to switch it on only if a communication demand occurs. The application of such an energy reduction strategy is numerous in the WSN. In this paper, the targeted scenario is the home multimedia network. As in the case of WSNs, in home multimedia networks, equipment, such as gateways, TV sets, PCs, and video game consoles, are used very sporadically and waste a large amount of energy even in standby mode. In order to solve this problem, a solution using a quasi-passive wake-up radio is proposed and thoroughly studied. The wake-up signal is sent by using an $802.11 \mathrm{~g}$ compliant emitter, commonly used in home multimedia equipment. Thus, there is no need of supplementary hardware.

The objective of this work is to propose a quasi-passive wake-up receiver (WuRx) for wireless sensor networks. Compared to the authors' previous work presented in [3], in this paper, a thorough study of the proposed WuRx in terms of identifier's choice, robustness, and evaluation of power saving compared to a classical technique was performed. A particular emphasis is made on home multimedia networks. Energy saving is enhanced at the expense

\section{黑 Springer}

(c) 2014 Hutu et al.; licensee Springer. This is an Open Access article distributed under the terms of the Creative Commons Attribution License (http://creativecommons.org/licenses/by/4.0), which permits unrestricted use, distribution, and reproduction in any medium, provided the original work is properly credited. 
of reduced sensitivity which may impact the overall system operating range. In a more general case, this problem can be tackled by either increasing the emitting antenna gain or the emitting power. Moreover, in order to indicate which particular node should wake up, an identification mechanism, using frequency fingerprints, is proposed and studied.

For example, it has been pointed out that more than half the energy consumption of a gateway would be saved if it is turned off during the night [4]. Moreover, the wakeup radio architectures such as the one proposed in this paper can be implementable as the majority of multimedia equipment has an orthogonal frequency-division multiplexing (OFDM) transmitter which is able to generate an identifier without any additional hardware.

More generally, efforts to reduce energy consumption are undertaken at different levels such as medium access protocols [5] by dynamically adapting the listen/sleep duty cycle. At the physical layer, efforts are made in order to propose energy-efficient waveforms and codes or optimized radio-frequency (RF) front ends. Another approach to increase energy efficiency is the cross-layer optimization, from physical to application layer [6].

All these efforts converge to the conclusion that at the physical layer, the control mechanisms must be created in order to activate or deactivate a part or the entire radio front end. In wireless sensor networks, in order to control the state of the radio front end, a second front end, with lower consumption compared to the main one, is proposed. The overlay network, composed by the low-energy front ends, will only convey the information on which set of nodes must communicate at a certain time.

This paper is divided as follows: Section 2 presents a state of the art of wake-up receivers. Section 3 proposes a new wake-up radio receiver that is validated via simulations in Section 4. Section 5 is dedicated to the comparison in terms of energy efficiency of a wake-up radio system and a classical power-saving mechanism implemented in 802.11 protocol. Section 6 summarizes the paper and highlights directions for future work.

\section{WuRx architectures and design considerations}

The sporadic use of the radio front ends highlights the need of a sleep state. This state corresponds to the smallest level of power consumption. To switch to an active state, a wake-up mechanism must exist. There are two kinds of wake-up strategies. First, synchronous protocols are needed to periodically activate the radio front ends. At regular time instants, the nodes probe the radiofrequency channel to determine whether another node wants to establish a communication, and the activation decision is taken at the node level. Such method supposes that global synchronization across all nodes in the wireless network is guaranteed. Second, remote nodes request a node to activate its main radio-frequency interface. In this case, the use of an auxiliary radio is needed.

The ideal case is to have zero DC power consumption in standby mode. This is why passive wake-up receivers have been proposed $[7,8]$. The key feature is that energy is received from a remote system. The majority of wakeup receivers are active. In this case, the autonomy of the entire network will be given by the power consumption in standby mode.

Radio-frequency wake-up systems can also be divided in two categories. The first group is represented by rectenna systems first presented in [8]. This kind of wake-up systems detects only a certain power level on a radio channel. Despite its very low power consumption, the main drawback is that it cannot make the difference between a noisy channel and a real wake-up signal, and consequently, its robustness in terms of false wake-ups is much degraded. The wake-up signal is transformed into a DC level by using charge pumps or voltage multipliers. In [8], a diode-based architecture is presented, and in [9], MOS transistors replace the diodes. The wake-up receivers are gathered into a second group of wake-up systems, which are able to receive, demodulate, and decode a wake-up signal.

In this category, the energy consumption is correlated to the type of modulation and there are two types of radio architectures. The first one is the classical homodyne architecture that transposes the received signal in the baseband signal [10]. The second type is the on-off keying $(\mathrm{OOK})$ modulation dedicated architecture, which is an envelope detector that recovers the digital signal as the amplitude of the radio-frequency signal. This last architecture is proposed in most of wake-up scenarios because of the low complexity and low energy consumption.

Table 1 presents a non-exhaustive state of the art of wake-up radio architectures based on OOK modulation.

The sensitivity and implicitly the range of the receiver are related to the DC power consumption. For higher sensitivity, a low-noise amplifier is needed and the DC power consumption increases from microamperes to milliamperes. Furthermore, the processing of the received signal, which is also an energy costly operation, is not taken into account here. Generally, an ultra-low power micro-controller is used in order to decode the received signal and to take the decision to whether wake up or not the main radio front end. In the architecture proposed in [3], the decision is taken at the RF level, and thus, the overall energy consumption is further reduced.

\section{Description of the wake-up radio architecture design}

Figure 1 presents a possible usage scenario of the proposed wake-up receiver. Suppose that all the sensors are in standby mode and the sink wants to communicate with 
Table 1 Wake up radio state of the art

\begin{tabular}{|c|c|c|c|c|c|}
\hline Reference & Frequency & DC power supply & Emitted RF power & Sensitivity & Range \\
\hline [8] & $433 \mathrm{MHz}$ & $1.07 \mu \mathrm{W}$ & $10 \mathrm{dBm}$ & $-51 \mathrm{dBm}$ & $10 \mathrm{~m}$ \\
\hline [11] & $868 \mathrm{MHz}$ & $2.4 \mu \mathrm{W}$ & $6.4 \mathrm{dBm}$ & $-71 \mathrm{dBm}$ & $300 \mathrm{~m}$ \\
\hline$[12]$ & $400 \mathrm{MHz}$ & $3.4 \mathrm{~mW}$ & $-2.17 \mathrm{dBm}$ & $-80.2 \mathrm{dBm}$ & - \\
\hline [13] & $2.4 \mathrm{GHz}$ & $2.59 \mathrm{~mW}$ & $0 \mathrm{dBm}$ & $-75 \mathrm{dBm}$ & $25 \mathrm{~m}$ \\
\hline [14] & $2.4 \mathrm{GHz}$ & - & $0 \mathrm{dBm}$ & - & $20 \mathrm{~m}$ \\
\hline$[15]$ & $3.1-10 \mathrm{GHz}$ & $4.5 \mathrm{~mW}$ & - & - & $10 \mathrm{~m}$ \\
\hline$[16]$ & $868 \mathrm{MHz}$ & $94.2 \mu \mathrm{W}$ & - & - & $3 \mathrm{~m}$ \\
\hline$[17]$ & $868 \mathrm{MHz}$ & $2.7 \mu \mathrm{W}$ & $10 \mathrm{dBm}$ & - & $40 \mathrm{~m}$ \\
\hline
\end{tabular}

the $i$ th node. In this case, the sink sends a frequency pattern which is the address associated to the node $i$. The wake-up radio of the $i$ th node switches on the power supply of the main data front end, and the communication begins while the rest of the sensors remain asleep. Once the communication is ended, the node deactivates the main radio interface and goes back into a standby mode where only the wake-up receiver is on.

Under the assumption that the sink has an OFDMcompliant transceiver, the wake-up signal is emitted by its classical data emitter, using a particular and predetermined sub-carrier pattern as identifier. The total number of sub-carriers is divided into a number $n$ of subgroups of adjacent sub-carriers, and in this way, $N$ sub-bands are formed. If there is spectral occupancy on a sub-band, this is associated to a logical 1 and the absence of signal on a sub-band to a logical 0 . In this way, $2^{N}$ possible identifiers can be formed.

In Figure 2, the block diagram of the proposed wake-up receiver is presented. The signal coming from the antenna is divided into two equal parts using a power divider. Then, the two versions are filtered by multi-band filters having complementary patterns, i.e., when the filter on the direct path allows passing the signal, on the complementary path, it will stop it and vice versa. Moreover, the filter on the direct path has the same frequency pattern as the identifier. As output, the two filters and two envelope detectors transform the radio-frequency signals into DC
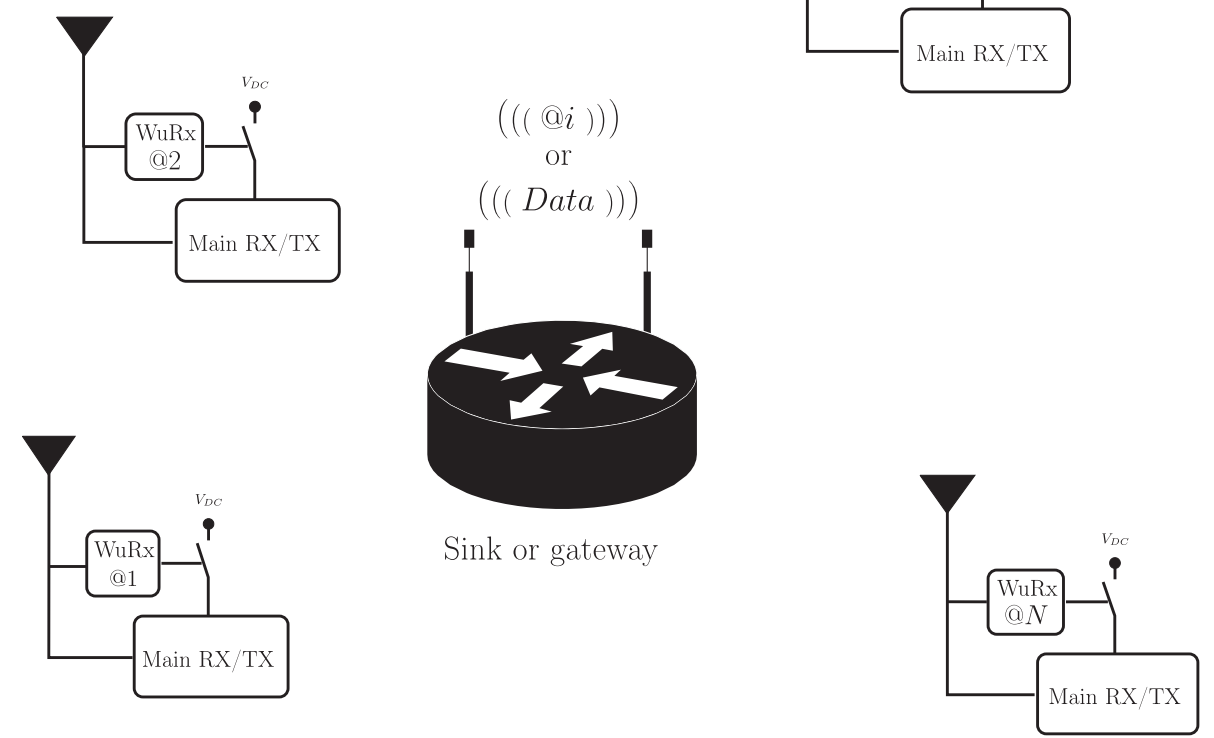

Figure 1 Wake-up mechanism. 


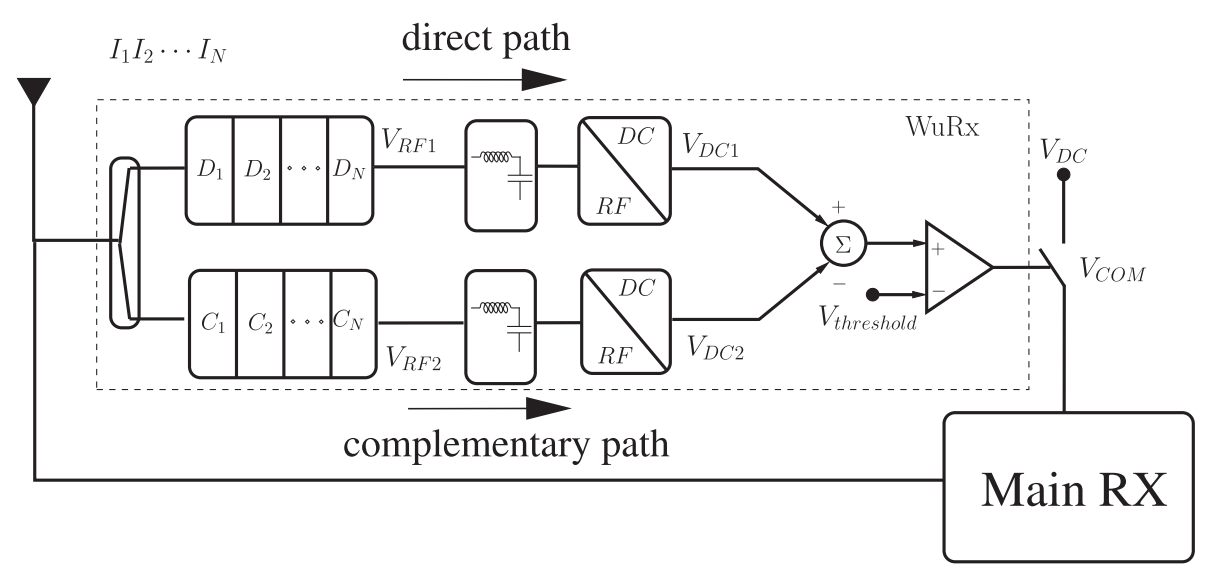

Figure 2 Block diagram of the proposed wake-up receiver.

levels, and a subtractor together with a comparator will toggle the $V_{\mathrm{COM}}$ output to a certain voltage level only if the identifier is received.

In Figure 3, the multi-band filters are arrays of bandpass filters having the $-3 \mathrm{~dB}$ bandwidth $\frac{\mathrm{BW}}{N}$, where $\mathrm{BW}$ is the total bandwidth of the OFDM signal. These filters should be reconfigurable in order to cover all identifier possibilities. The central frequency of the $j$ th filter is

$$
f_{j}=f_{0}+\frac{(2 j-1)-N}{2 N} \mathrm{BW}, j \in\{1, \cdots, N\},
$$

where $f_{0}$ is the central frequency of the channel used to sent the identifier.

As for the OFDM sub-bands, let us assume that if the direct or complementary filter on one of the $N$ sub-bands is a band-pass filter, this is associated to a logical 1, and if it has a stop-band behavior on a sub-band, this is associated to a logical 0 . Then for one equipment, the associated identifier will have the same code as the direct path filter. The triplet: identifier $\left(I_{K}\right)$, direct path filter $\left(D_{K}\right)$, and complementary path filter $\left(C_{K}\right)$ can be modelled as follows:
$\left\{\begin{array}{l}I_{K} \in\left\{I_{N} \cdots I_{k} \cdots I_{1} \text {; with } I_{k} \in\{0,1\}, \forall k=1 \cdots N\right\} \\ D_{K} \in\left\{D_{N} \cdots D_{k} \cdots D_{1} ; \text { with } D_{k} \in\{0,1\}, \forall k=1 \cdots N\right\} \\ C_{K} \in\left\{C_{N} \cdots C_{k} \cdots C_{1} \text {; with } C_{k} \in\{0,1\}, \forall k=1 \cdots N\right\}\end{array}\right.$.

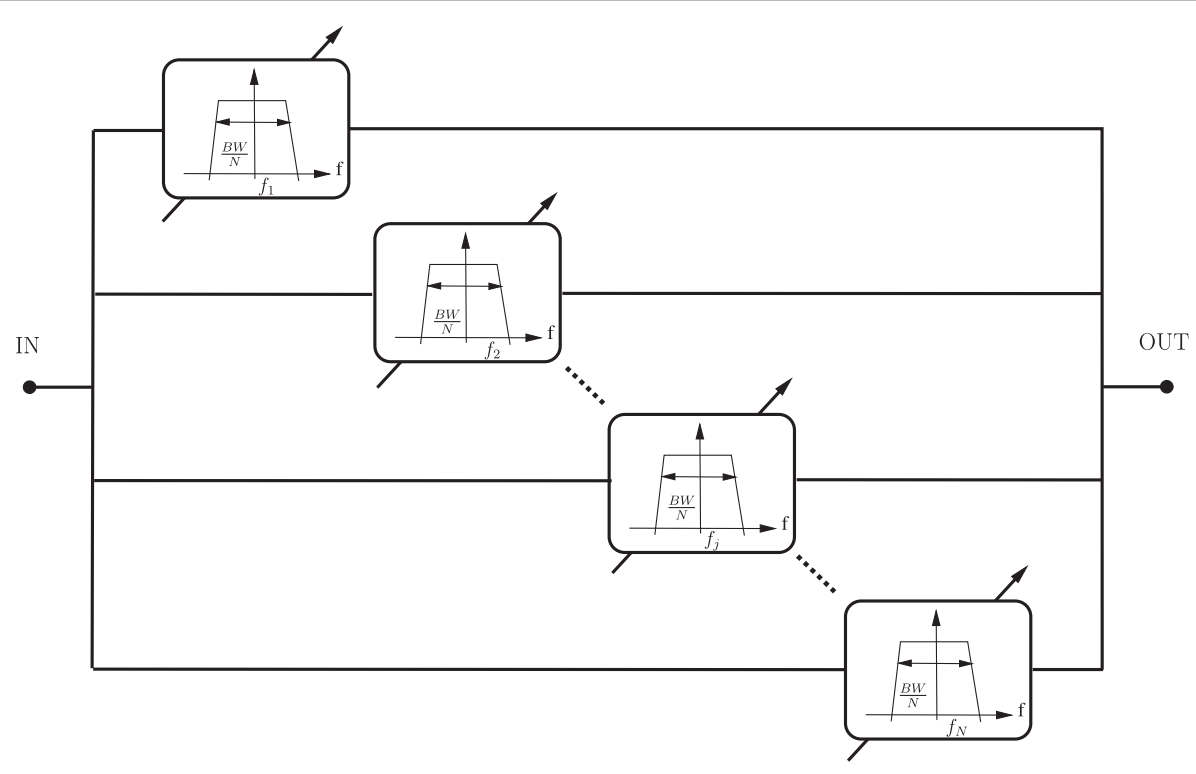

Figure 3 Block diagram of one of the bank filters. 
Note that not all the $2^{N}$ possible combinations can be used as identifiers. One can see that, for example, in the case $N=4$, the identifier @1010 will address both the node having 1010 as the identifier and the node having 1011 as identifier. This can be subject to false wakeups and consequently will increase the overall energy consumption. An appropriate choice of triplet identifier, direct path filter, and complementary path filter must be done in order to avoid such ambiguity. Three conditions must accomplish a triplet $\left\{I_{K}, D_{K}, C_{K}\right\}$ in order to be considered as viable. These conditions are given in system (3):

$$
\left\{\begin{array}{l}
\sum_{L=1}^{N} D_{K, L} \cdot I_{K, L}-\sum_{L=1}^{N} C_{K, L} \cdot I_{K, L}>0 \\
\sum_{L=1}^{N} D_{K, L} \cdot \Pi-\sum_{L=1}^{N} C_{K, L} \cdot \Pi \leqslant 0 \\
\sum_{L=1}^{N} D_{K, L} \cdot I_{T, L}-\sum_{L=1}^{N} C_{K, L} \cdot I_{T, L} \leqslant 0 ; \forall T \in\left\{1 \cdots 2^{N}-2\right\} \backslash K
\end{array}\right.
$$

where $\Pi$ is a classical $802.11 \mathrm{~g}$ signal having the same bandwidth as the 1111 identifier. This is necessary to overcome the problem of $802.11 \mathrm{~g}$ signals emitted in the proximity of the wake-up receiver which, in the case of the home networking scenarios, is high. The indexes $K$ and $T$ are used to express a certain identifier and together with its associated direct and complementary path filters and the index $L$ are used to express one of the $N$ sub-bands of an identifier.

These three conditions are established supposing that for one sensor, the direct and complementary filters are fixed. The first condition in (3) states that, in the presence of the assigned identifier, the energy at the direct filter's output must be greater than the energy at the output of the complementary path filter. The second constraint is set in order to avoid wake-ups in the presence of a classical OFDM signal. In this case, the energy at the output of the direct path filter should be less than or equal to the energy at the output of the complementary path filter. The third condition states that for any other identifier, other than the assigned one, the energy at the direct output filter must be less than or equal to the energy at the output of the complementary path filter.

These conditions will give all the viable triplets $I_{K} D_{K}, C_{K}$. For example, in the case $N=4$ sub-bands, there are only six possible combinations respecting these three conditions. The available combinations are given in Table 2.

This drastically reduces the number of addressed sensors. In order to circumvent this problem, the solution could be to increase the number of sub-bands. However, filters with high quality factor must be implemented. Therefore, a compromise between the number of addressed sensors and the technological constraints
Table 2 Possible triplet identifier-direct path filter-complementary path filter for four sub-bands

\begin{tabular}{lcc}
\hline Identifier & Direct path filter & Complementary path filter \\
\hline 0011 & 0011 & 1100 \\
0101 & 0101 & 1010 \\
0110 & 0110 & 1001 \\
1001 & 1001 & 0110 \\
1010 & 1010 & 0101 \\
1100 & 1100 & 0011 \\
\hline
\end{tabular}

should be found. For example, for home networking applications, this set of six possible addresses could be sufficient.

\section{Simulation results}

To validate the behavior of the proposed wake-up receiver, a series of circuit system co-simulations using Agilent's ADS software were performed. The identifiers were generated by modifying the baseband part of a $802.11 \mathrm{~g}$ source, using a $20 \mathrm{MHz}$ channel around a $2.45 \mathrm{GHz}$ carrier frequency and grouping the sub-carriers in $N=4$ equally spaced subsets. A total number of 64 sub-carriers with $312.5 \mathrm{KHz}$ spacing between them were used. The identifier $I_{4} I_{3} I_{2} I_{1}=\mathbf{0 1 0 1}$ is presented in Figure 4 .

The multi-band filters have been simulated using filter banks, each band-pass filter having $5 \mathrm{MHz}$ of $-3 \mathrm{~dB}$ bandwidth with an insertion loss of less than $1 \mathrm{~dB}$. The out-ofband rejection is considered at $-40 \mathrm{~dB}$. The needed quality factor of the filter is 480 which cannot be obtained by classical passive filters. Nevertheless, in order to overcome this problem, one solution is to use $10 \mathrm{MHz}$ sub-bands instead of $5 \mathrm{MHz}$ and cavity filters as the ones presented in [18] with measured quality factors greater than 300.

The matching circuits, the direct and complementary path envelope detectors, the subtractor, and the Schmidt trigger have been simulated using the schematic depicted in Figure 5.

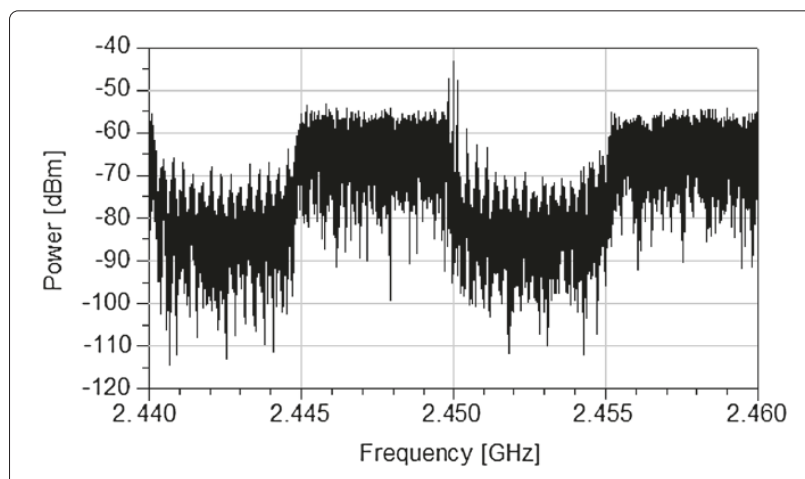

Figure 4 Example of 0101 identifier. 


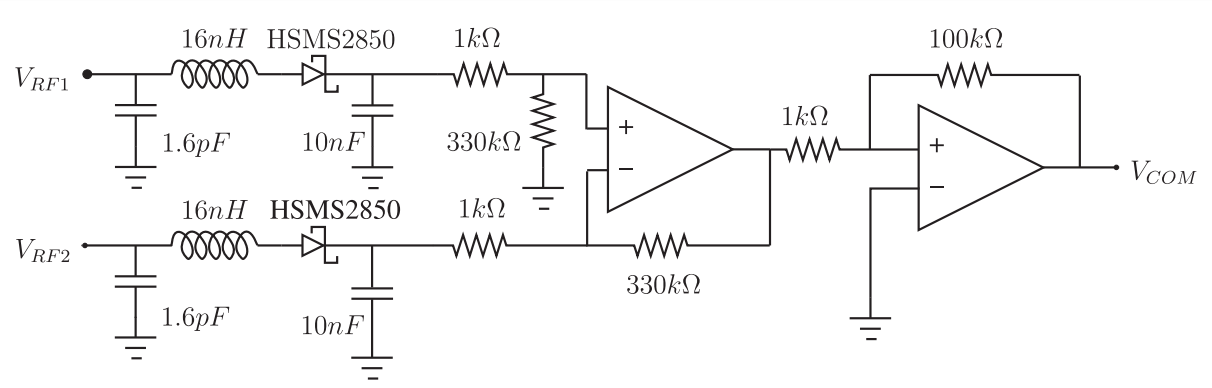

Figure 5 The matching circuits, envelope detectors, subtractor, and Schmidt trigger.

One of the key points of the proposed architecture is the global sensitivity given by the envelope detector sensitivity. Generally, a zero polarization Schottky diode is used, and in this case, the sensitivity is defined by the lowest detected RF power level which gives an $8 \mathrm{~dB}$ output signal-to-noise ratio [19].

Another important parameter of the Schottky diode is its responsivity defined as the DC voltage at the output of the envelope detector function of the input RF power level. The simulations were conducted using HSMS2850 diodes having $-50 \mathrm{dBm}$ sensitivity and a $60 \mathrm{mV} / \mu \mathrm{W}$ responsivity. Under these conditions, the DC output voltage varies from $550 \mu \mathrm{V}$ to $420 \mathrm{mV}$. For low input power levels, the DC voltage at the output of the envelope detector may not be sufficient to drive the subtractor's inputs. In a real implementation, voltage multipliers could be designed in order to overcome this potential problem. The voltage multipliers are passive elements formed by zero polarization Schottky diodes and capacitors, and consequently, there is will be no influence on the overall wake-up radio energy consumption.

The outputs of the two envelope detectors are connected to the inputs of the subtractor which is simulated using an operational amplifier model. The Schmidt trigger is also simulated using the same model of operational amplifier. As the two op amps are the only active elements in the circuit, the overall power consumption of the proposed wake-up receiver is estimated at $1.18 \mu \mathrm{A}$ [20], which is near to the lowest power consumption in the state of the art for the RF part when no decision circuit is taken into account.

In Figure 6, the outputs of the direct path and complementary path envelope detector are presented. In this case, the identifier is sent for $400 \mathrm{~ns}$ and the distance between the emitter and the wake-up receiver is set up at $20 \mathrm{~m}$.

A range estimation of the proposed wake-up receiver can be calculated. To do so, it is considered that the receiving antenna has enough gain to compensate the insertion losses of the multi-band filters and the power divider used to convey the received signal to the filters. The minimum received signal is approximated by the Schottky diode sensitivity. Considering the application, the first approach is to model the propagation channel as an indoor one having a path loss exponent between 1.6 and 3 and a shadowing margin equal to $5 \mathrm{~dB}$. It is also considered that the identifier is emitted with a maximum equivalent isotropically radiated power (EIRP) of $20 \mathrm{dBm}$ and the receiving antenna is omnidirectional with $2 \mathrm{dBi}$ gain. Under these conditions, the estimated range is between 8 and $48 \mathrm{~m}$, depending on the harshness of the radio channel. In our case, the proposed wake-up receiver, by its quasi-passive architecture, favors the energy saving at the expense of the reduced sensitivity and consequently reduced operating

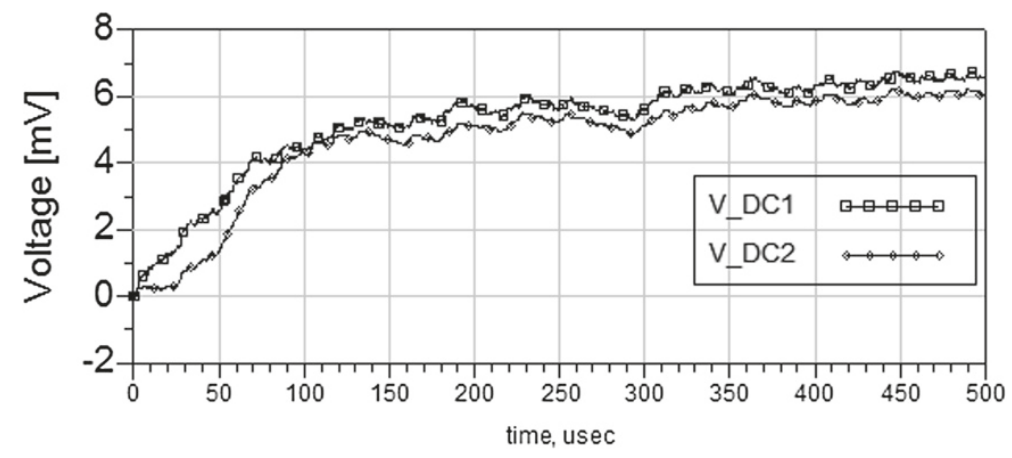

Figure 6 The envelope detector outputs. 
range. In this way, the overall autonomy of a wireless sensor network in which sporadic data exchange occurs, can be increased.

The delay inserted by the wake-up radio is another important parameter since it will impact the overall WSN latency. Generally, the delay inserted by a common microcontroller to take the decision is approximatively tenths of milliseconds. Figure 7 depicts the time latency of our proposed wake-up receiver when the received power varies between -50 and $-10 \mathrm{dBm}$. As it can be seen, the time latency increases with the decrease of the received power level, and this is due to the charge time of the capacitors in the envelope detectors which is slower for low power levels. The start time on this curve corresponds to the start of transmission of the identifier. Nevertheless, the maximum delay is around $100 \mu \mathrm{s}$.

In order to verify the robustness of the proposed architecture, the worst-case scenario, in which a second transmitter emits a classical OFDM signal on the same channel as the identifier, was simulated. Furthermore, the power levels of the two signals are considered identical. In the left part of Figure 8, the frequency shape of the signal received by the wake-up radio is given. As it can be noticed, the identifier is 'flooded' by the interfering signal. Despite this, after passing through the two filters having a complementary structure, the action of the interfering signal is canceled and the output of the comparator toggles to a high voltage level, as it can be seen in the right part of Figure 8.

Moreover, suppose that the same signal composed by 0101 and a classical OFDM signal are received by the equipment having 0110 as direct path filter and 1001 as complementary path filter. Figure 9 presents the subtractor's output voltage after the transient period for different identifier power levels. If the identifier's power level remains below $-17 \mathrm{dBm}$ when the OFDM signal is at $-20 \mathrm{dbm}$, then no false wake-up will occur. Indeed, the subtractor's maximum output voltage remains below the threshold level defined by the Schmidt trigger.

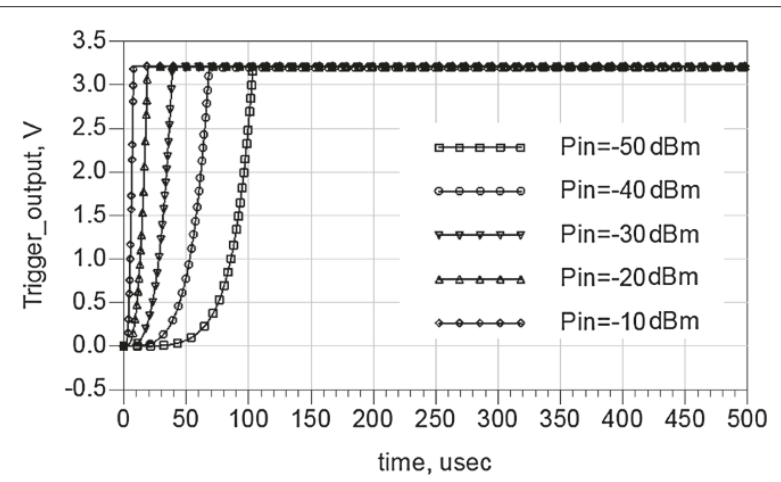

Figure 7 The Schmidt trigger output for various values of the identifier's input power.

\section{Evaluation of the potential power saving}

This section compares the power saving when using a wake-up receiver and the power saving when using the classical 802.11 power save mode (PSM). The power save mode is designed for ad hoc networks and is a simple protocol similar to other mechanisms, such as automatic power save delivery (APSD) and power save multi-poll (PSMP) [21].

The PSM mode allows nodes to turn into two different states, active and doze states (equivalent to sleeping mode of a radio front end), and because there is no supervising identity, the network is synchronized. This means that there is a time interval called ad hoc traffic indication message (ATIM) window where all the nodes are awake. A node which wants to communicate sends a target beacon during the target beacon transmission time (TBTT). The second part of the ATIM window is about organizing the transmission until the next beacon frame.

The transmission mechanism is as follows: at the transmitter side, if a node wants to transmit data, it has to send an ATIM message within the ATIM window period. If the ATIM message has been acknowledged, then the transmitter will send the data after a random time. At the receiver side, if a node receives an ATIM message, it means that it is involved in a transmission during the next beacon interval. The receiver will send an ACK message and will stay awake until the next TBTT. If a node does not send or receive an ATIM message, it means that it can turn to doze state during the next beacon interval and then wake up for the next TBTT.

In the case of using a wake-up receiver, compared to the 802.11 power save mode, an asynchronous mechanism is implemented. If a node wants to communicate to another node, it will send the distant node address as a frequency pattern. The corresponding node will wake up and the data transfer will begin. In order to be more energy efficient, it has been considered that when the communication between the two nodes ends, the receiver's node radio front end goes into off mode, i.e., the node's energy consumption is only the energy consumption of the wake-up receiver.

In order to make this comparison, a simple scenario of a point-to-point transmission between two nodes STA $A$ and STA $B$ is considered. Depending on the moment when one node (STA $A$ ) decides to send data to the other node (STA $B$ ), there are two particular cases presented in the following. The first one, the most probable one, is when STA $A$ decides to send a message to the STA $B$ outside an ATIM window (the case marked WuRx case (a) in Figure 10) and the case when STA $A$ decides to send a message to STA $B$ during the ATIM window (case $W u R x$ (b) in Figure 10).

Figure 10 shows the power $P_{\mathrm{ON}}$ consumed by the main radio front end, the power consumption $P_{\text {STDB-WIFI }}$ when 

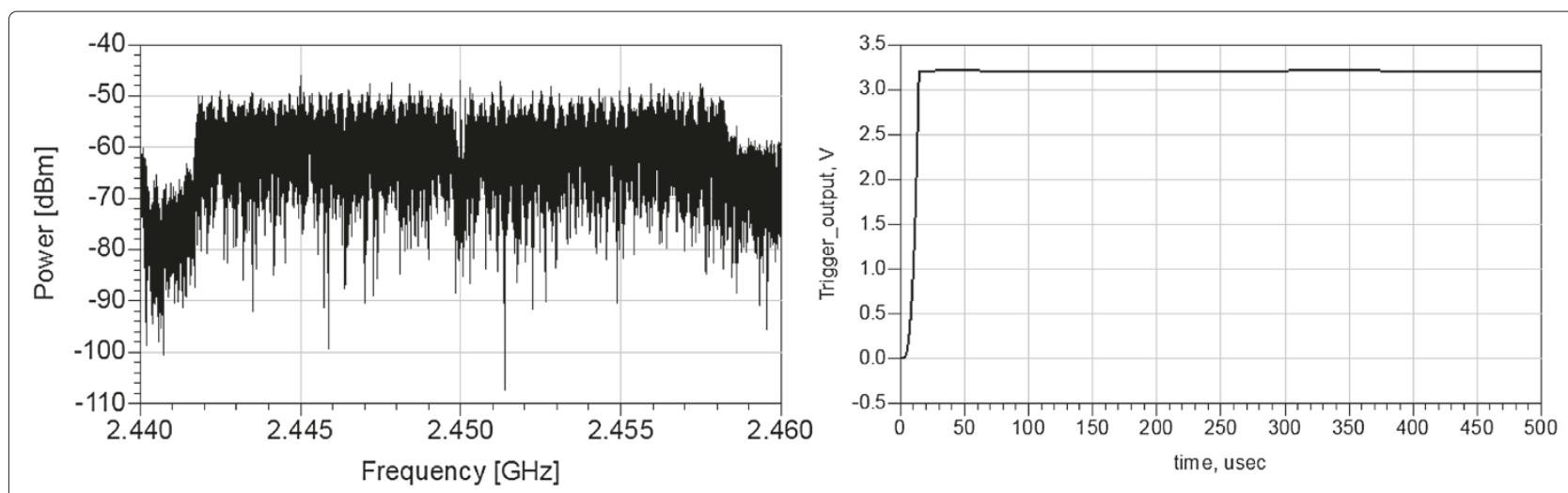

Figure 8 OFDM signal interferer and the $V_{\text {COM. }}$. A classical OFDM signal interferer superposed to 0101 identifier (left) and $V_{\text {COM }}$ voltage in this particular case (right).

one node is in standby mode, the power $P_{\mathrm{ID}}$ consumed by the STA $A$ when sending the identifier in the case of using the wake-up radio, and the power consumption $P_{\text {STDB-WuRx }}$ of the STA $B$ when the wake-up radio is used. The Turn on $R X$ interval is the time interval when the STA $B$ receiver passes from the off state to receive state. As seen from Table 3, this time interval is mainly given by the crystal oscillator turn-on time.

For a time observation window $T$, the overall energy consumption in the case of using PSM is

$$
\begin{aligned}
\varepsilon_{\mathrm{PSM}}= & P_{\mathrm{SB}-\mathrm{WIFI}}\left(T-2 T_{\mathrm{ATIM}}-T_{\mathrm{DATA}}\right) \\
& +P_{\mathrm{ON}}\left(2 T_{\mathrm{ATIM}}+T_{\mathrm{DATA}}\right)
\end{aligned}
$$

where $T_{\text {ATIM }}$ is the ATIM window period, $T_{\text {DATA }}$ is the time period needed by STA $A$ to send the data to STA $B$. When using the wake-up radio, it can be seen that the overall energy consumption is

$$
\begin{aligned}
\varepsilon_{\mathrm{WuRx}}= & P_{\mathrm{SB}-\mathrm{WuRx}}\left(T-T_{\mathrm{DATA}}-T_{\mathrm{toRx}}-T_{\mathrm{ID}}\right) \\
& +P_{\mathrm{ID}} T_{\mathrm{ID}}+P_{\mathrm{ON}} T_{\mathrm{Data}}+P_{\mathrm{toRx}} T_{\mathrm{toRx}}
\end{aligned}
$$

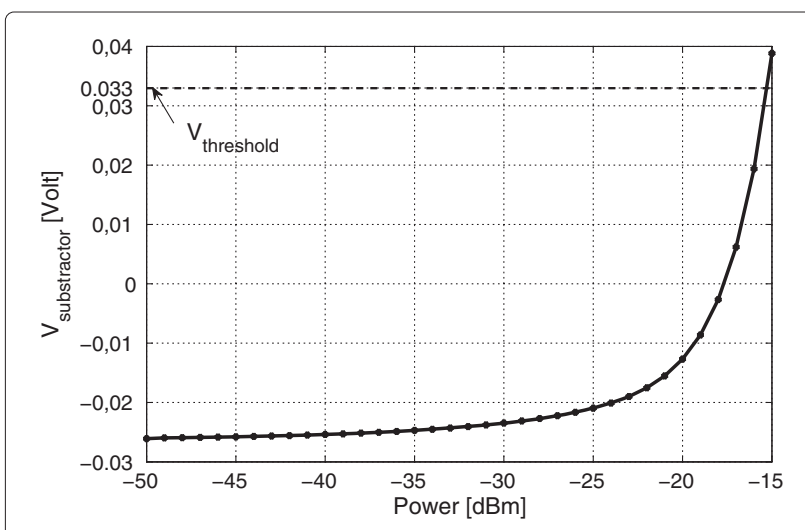

Figure 9 The variation of the subtractor's output voltage. where $T_{\mathrm{ID}}$ is the time needed to send the identifier by STA $A$ and $T_{\text {toRx }}$ is the time needed by STA $B$ to pass from the off state to receive state.

From the last two equations, the comparison between the two power save mechanisms can be performed by comparing the two global energies. It can be seen that there are cases where the power save mode is more efficient than the wake-up radio approach especially when the energy spent to send the identifier and activate the main radio front end in the wake-up case is greater than the energy spent in standby in the case of PSM, as it can be seen from Equation (6).

$$
P_{\mathrm{SB}-\mathrm{WIFI}} T_{\mathrm{SB}-\mathrm{WIFI}} \geq P_{\mathrm{ID}} T_{\mathrm{ID}}+P_{\mathrm{toRx}} T_{\mathrm{toRx}},
$$

where $T_{\mathrm{SB}-\mathrm{WIFI}}=T-2 T_{\mathrm{ATIM}}-T_{\mathrm{DATA}}$.

The last inequality stands if $P_{\mathrm{SB}-\mathrm{WIFI}} \gg P_{\mathrm{SB}-\mathrm{WuRx}}$ and the terms $P_{\mathrm{SB}-\mathrm{WuRx}} T, P_{\mathrm{SB}-\mathrm{WuRx}} T_{\mathrm{DATA}}$, and $P_{\mathrm{SB}-\mathrm{WuRx}} T_{\mathrm{toRx}}$ are negligible compared to $P_{\mathrm{SB}-\mathrm{WIFI}} T, P_{\mathrm{SB}-\mathrm{WIFI}} T_{\mathrm{DATA}}$, and $P_{\text {SB-WIFI }} 2 T_{\text {ATIM }}$, respectively.

From inequality (6), a minimum bound for the standby time in power save mode can be given. Beyond this bound, the power save mode became more energy efficient than the wake-up radio approach:

$$
T_{\mathrm{SB}-\mathrm{WIFI}} \geq \frac{P_{\mathrm{ID}} T_{\mathrm{ID}}+P_{\mathrm{toRx}} T_{\mathrm{toRx}}}{P_{\mathrm{SB}-\mathrm{WIFI}}} .
$$

In order to numerically illustrate these equations, the proposed wake-up receiver and Maxim's MAX 2830 radio transceiver were considered as practical case. The MAX 2830 transceiver [22] is designed specifically for 2.4 to 2.5 $\mathrm{GHz} 802.11 \mathrm{~b} / \mathrm{g}$ WLAN applications. It is a single-chip, low-power, direct conversion, zero-IF transceiver integrating all circuitry required to implement $\mathrm{RF}$ transceiver functions. In Table 3, the different parameters used in the energy model extracted from the MAX 2830 datasheet are gathered. 


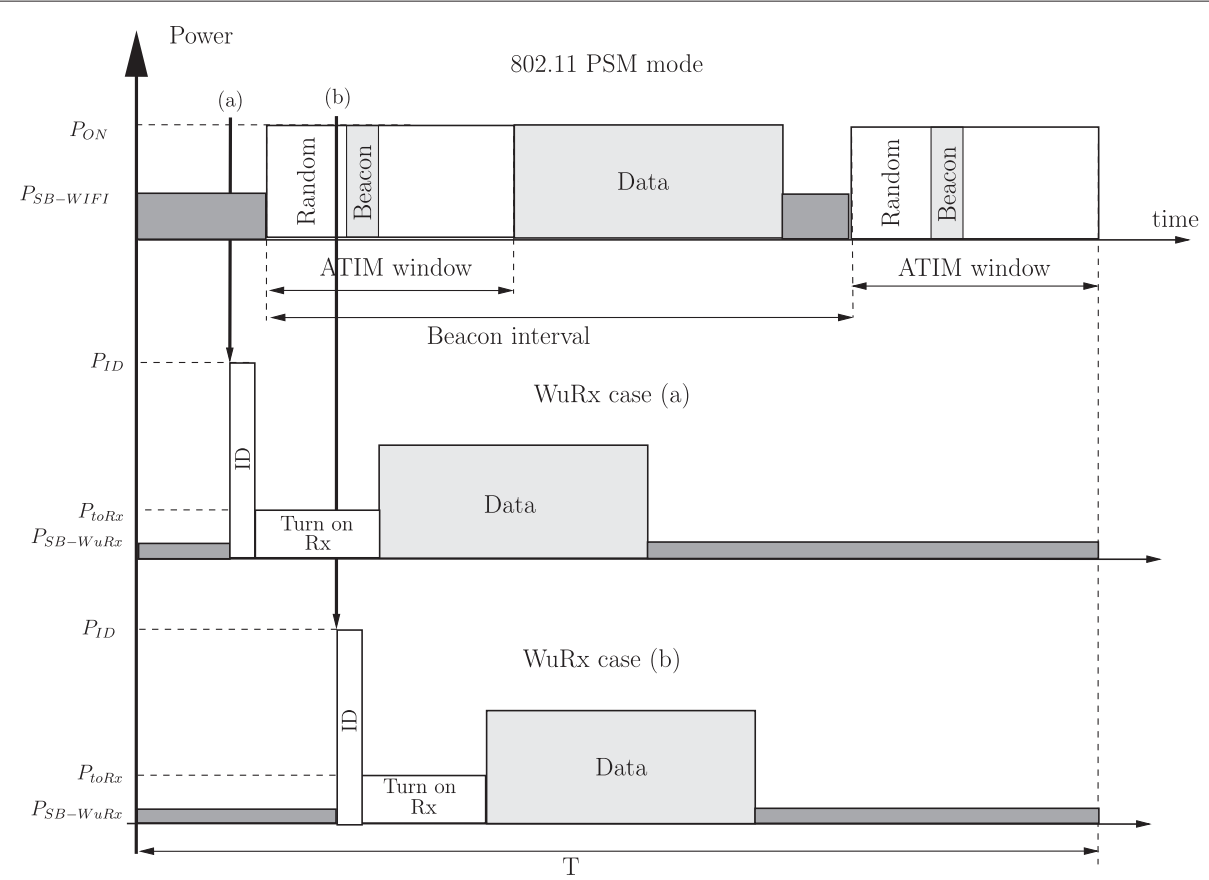

Figure 10 PSM global energy consumption versus WuRx global energy consumption.

As it was seen previously, the time to send the identifier $T_{\mathrm{ID}}=400 \mu \mathrm{s}$, and the power consumed by the wake-up receiver is $400 \mathrm{nW}$. In this case, according to inequality (7), the standby time in power save mode should be less than $5.27 \mathrm{~ms}$ in order to spend less energy in power save mode than in the case of using the proposed wake-up radio. Considering the typical values of the ATIM window (40 $\mathrm{ms})$ and the typical beacon interval $(102.5 \mathrm{~ms})$, the

Table 3 Parameters for modelling the energy consumption using a MAX2830 transceiver

\begin{tabular}{|c|c|c|}
\hline Variable & Description & Value \\
\hline$P_{\mathrm{ID}}$ & Power consumption in transmission mode & $800 \mathrm{~mW}$ \\
\hline$P_{\mathrm{ON}}$ & Power consumed in reception mode & $167.4 \mathrm{~mW}$ \\
\hline$P_{\text {SB-WIFI }}$ & Power consumption in standby mode & $75.6 \mathrm{~mW}$ \\
\hline$P_{\text {sleep }}$ & $\begin{array}{l}\text { Power consumed by the radio interface } \\
\text { in sleep mode }\end{array}$ & $75.6 \mathrm{~mW}$ \\
\hline$P_{\text {Turn-On }}$ & $\begin{array}{l}\text { Power consumed by the radio interface } \\
\text { in shutdown mode }\end{array}$ & $54 \mu \mathrm{W}$ \\
\hline$P_{\text {oscil }}$ & $\begin{array}{l}\text { Power consumed by the frequency } \\
\text { synthesizer block }\end{array}$ & $75.6 \mathrm{~mW}$ \\
\hline$T_{\text {Backoff }}$ & Time of backoff & $67.5 \mu \mathrm{s}$ \\
\hline$T_{\text {Turn-On }}$ & $\begin{array}{l}\text { Time to pass from off mode to } \\
\text { shutdown mode }\end{array}$ & $60 \mu \mathrm{s}$ \\
\hline$T_{\text {toRx }}$ & $\begin{array}{l}\text { Time to settle the frequency of the } \\
\text { crystal oscillator }\end{array}$ & $1 \mathrm{~ms}$ \\
\hline$T_{\text {RX-On }}$ & $\begin{array}{l}\text { Time to pass from standby mode to } \\
\text { receive mode }\end{array}$ & $2 \mu \mathrm{s}$ \\
\hline
\end{tabular}

observation interval is $T=147.77 \mathrm{~ms}$. One can say that using a wake-up radio is a more energy-saving option compared to using the PSM for ratios $T_{\mathrm{SB}-\mathrm{WIFI}} / T$ greater than $3.56 \%$. Therefore, for sporadic data transmission, wake-up radio usage will always be more energy efficient than the power save mode.

\section{Conclusions}

In this paper, a wake-up radio circuit was proposed and validated by circuit system co-simulations. The identification of the targeted sensor to wake up is done by sending a particular frequency spectrum, shaped using a classical OFDM spectrum. This approach is suitable for home multimedia networks in which most of the equipment have a 802.11 OFDM-compliant transceiver. A theoretical study of the possible combinations - identifier, direct path filter, and complementary path filter - is also presented. The advantage of this architecture lies in the simplicity of the decision circuit, which consists of two multi-band filters. This implies a reduction of the energy consumption of the entire wake-up radio because of the absence of a more sophisticated decision circuit based on a microcontroller, for example, and also because of the absence of a dedicated emitter for the transmission of the wake-up signal. Complementary studies are undertaken in order to quantify the robustness of the proposed architecture with respect to false wake-ups. Moreover, a prototype will be manufactured in order to have measurements on range, delay, and actual energy consumption. 


\section{Competing interests}

The authors declare that they have no competing interests.

\section{Acknowledgements}

This work was funded by the French FUl program: Econhome. The authors wish to thank the partners for their support and collaboration in this project.

Received: 23 April 2014 Accepted: 20 October 2014

Published: 28 October 2014

\section{References}

1. K Martinez, JK Hart, R Ong, Environmental sensor networks. Computer. 37(8), 50-56 (2004)

2. C Buratti, A Conti, D Dardari, R Verdone, An overview on wireless sensor networks technology and evolution. Sensors. 9(9), 6869-6896 (2009)

3. F Hutu, A Khoumeri, G Villemaud, J-M Gorce, in Proceedings of the 2014 IEEE Radio and Wireless Symposium. Wake-up radio architecture for home wireless networks (Newport Beach, California, USA, 19-23 Jan. 2014) pp. $256-258$

4. http://www.systematic-paris-region.org/fr/projets/econhome-v2 (2014)

5. Dam van, $\mathrm{K}$ Langendoen, in International Conference on Embedded Networked Sensor Systems. An adaptive energy efficient MAC protocol for wireless sensor networks (Los Angeles, California, USA, 5-7 Nov. 2003), pp. $171-180$

6. G Miao, N Himayat, YG Li, A Swami, Cross-layer optimization for energy-efficient wireless communications: a survey. Wireless Comm. Mobile Comput. 9(4), 529-542 (2009)

7. L Gu, JA Stankovic, in Real-Time and Embedded Technology and Applications Symposium, 2004. Proceedings. RTAS 2004. 10th IEEE. Radio-triggered wake-up capability for sensor networks (Toronto, Canada, 25-28 May 2004), pp. 27-36

8. L Gu, JA Stancovic, Radio-triggered wake-up for wireless sensor networks. R. Time Syst. 29, 157-182 (2005)

9. H Kim, H Cho, Y Xi, M Kim, S Kwon, J Lim, Y Yang, CMOS passive wake-up circuit for sensor network applications. Microw. Opt. Tech. Lett. 52(3), 597-600 (2010)

10. NM Pletcher, S Gambini, J Rabaey, A $52 \mu \mathrm{W}$ wake-up receiver with -72 $\mathrm{dBm}$ sensitivity using an uncertain-IF architecture. IEEE J. Solid State Circ. 44(1), 269-280 (2009)

11. C Hambeck, S Mahlknecht, T Herndl, in IEEE International Symposium on Circuits and Systems. A $2.4 \mu \mathrm{W}$ wake-up receiver for wireless sensor nodes with $-71 \mathrm{dBm}$ sensitivity (Rio de Janeiro, Brasil, 15-18 May 2011)

12. J Liu, C Li, L Chen, Y Xiao, J Wang, H Liao, R Huang, in 2011 Proceedings of the ESSCIRC (ESSCIRC). An ultra-low power $400 \mathrm{MHz}$ OOK transceiver for medical implanted applications (Helsinki, Finland, 12-16 Sept 2011), pp. $175-178$

13. M Vidojkovic, X Huang, P Harpe, S Rampu, C Zhou, L Huang, K Imamura, B Busze, F Bouwens, M Konijnenburg, J Santana, A Breeschoten, J Huisken, G Dolmans, H de Groot, in 2011 IEEE International Solid-State Circuits Conference Digest of Technical Papers (ISSCC). A 2.4GHz ULP OOK single-chip transceiver for healthcare applications (San Francisco, California, USA, 20-24 Feb. 2011), pp. 458-460

14. R de Francisco, Y Zhang, in 2011, IEEE Wireless Communications and Networking Conference (WCNC). An interference robust multi-carrier wake-up radio (Cancun, Mexico, 28-31 March 2011), pp. 1265-1270

15. R Hamdi, A Bounif, A Desmarais, D Deslandes, F Nabki, in New Circuits and Systems Conference (NEWCAS), 2011 IEEE 9th International. A low-power OOK ultra-wideband receiver with power cycling (Bordeaux, France, 26-29 June 2011). pp. 430-433

16. V Rosello, J Portilla, T Riesgo, in IECON 2011 - 37th Annual Conference on IEEE Industrial Electronics Society. Ultra low power FPGA-based architecture for wake-up radio in wireless sensor networks (Melbourne, Australia, 7-10 Nov 2011), pp. 3826-3831

17. GU Gamm, M Sippel, M Kostic, LM Reindl, in International Conference on Intelligent Sensors, Sensor Networks and Information Processing. Low power wake-up receiver for wireless sensor nodes (Brisbane, Australia, 7-10 Dec 2010)

18. X Liu, LPB Katehi, WJ Chappell, D Peroulis, High- tunable microwave cavity resonators and filters using SOI-based RF MEMS tuners. IEEE J. Microelectromech. Syst. 19(4), 774-784 (2010)
19. X Fan, X Pei, X Xiong, in 2011 International Conference on Electronics and Optoelectronics (ICEOE). Zero bias Schottky diodes use in high performance detection circuits, vol. 3 (Dalian, China, 29-31 July 2011)

20. http://www.intersil.com/content/dam/Intersil/documents/is/2/is/2819495.pdf (2014)

21. IEEE, IEEE Standard for Information Technology - Telecommunications and information exchange between systems - local and metropolitan area networks - specific requirements - part 11: wireless LAN medium access control (MAC) and physical layer (PHY) specifications - Redline, http://ieeexplore.ieee.org/stamp/stamp.jsp?arnumber=6522432 (2012)

22. http://datasheets.maximintegrated.com/en/ds/MAX2830.pdf (2014)

doi:10.1186/1687-1499-2014-177

Cite this article as: Hutu et al: A new wake-up radio architecture for wireless sensor networks. EURASIP Journal on Wireless Communications and Networking 2014 2014:177.

\section{Submit your manuscript to a SpringerOpen ${ }^{\mathcal{O}}$ journal and benefit from:}

- Convenient online submission

- Rigorous peer review

- Immediate publication on acceptance

- Open access: articles freely available online

- High visibility within the field

- Retaining the copyright to your article

Submit your next manuscript at $>$ springeropen.com 